\title{
Diet and weight loss apps: a review of user ratings, quality of nutritional information, inclusion of behaviour change techniques and personalised nutrition features
}

Effective weight loss interventions are needed to address the high levels of obesity and overweight which persist in the UK Smartphone mobile applications (apps) could offer cost-effective, personalised weight-loss and dietary advice to a broad population whilst utilising technological features such as barcode scanners to ease the burden of dietary and physical activity reporting ${ }^{(1)}$. Many health and fitness apps are available, but studies assessing efficacy are very limited and reviews indicate many apps are of low quality despite scoring highly in the app store ratings ${ }^{(2)}$. In the absence of regulatory standards, consumers need guidance to help them select apps with evidence-based content to support behaviour change, healthy eating habits and successful weight loss if appropriate, in addition to being engaging and appealing to use.

This study aimed to assess the evidence-based features incorporated into popular English language apps (including the quality of any nutritional information, the use of behaviour change techniques (BCTs) and personalisation) and to investigate the correlation between these features and app store star ratings.

A systematic search of the Google Play store resulted in a final sample of $n=30$ free apps meeting the criteria. Selected apps were downloaded onto a Samsung Galaxy A21s smartphone and reviewed using a combination of validated tools and self-developed checklists.

According to the MARS ${ }^{(3)}$ five-point scale, the selected apps were of acceptable quality (Mean [SD] = 3.0 [0.7]. Overall nutritional information content scores were low $(\mathrm{Md}(25 \%, 75 \%)=4.0(1.0,11.0))$ out of 25 , with less than half of apps $(47 \%)$ calculating energy requirements and only $32 \%$ calculating a BMI for the user. Only $20 \%$ of apps mentioned saturated fat guidelines and $17 \%$ included salt, fibre and fruit and vegetable recommendations. On average, apps included 9 BCTs with goal setting (behaviour) and self-monitoring of behaviour occurring most frequently. Whilst $77 \%$ of apps allowed input personalisation, less than half provided a personalised daily calorie requirement (47\%), food logging (43\%) or a BMI (30\%) and only 2 apps out of 30 offered personalised messaging. Mean scores for quality, nutritional information, BCTs and personalisation were positively associated with each other ( $\mathrm{r} / \mathrm{rho} 0.37$ to $0.86, \mathrm{p}<0.05)$, but Google Play star rating was not correlated with any of the other variables.

Overall, the apps studied were of moderate quality and although some apps incorporated evidence-based features, many scored poorly. Since app store popularity ratings do not reflect this variation, this type of review offers users and health care professionals a comprehensive guide to inform choice and highlights the need for technology literacy training to allow users to make informed choices about the tools they access to maximise their chances of achieving health behaviour change.

\section{References}

1. Coughlin SS, Whitehead M, Sheats JQ et al. (2015) Jacobs J Food Nutr 2(3), 021.

2. Azar KMJ, Lesser LI, Laing BY et al. (2013) Am J Prev Med 45, 583-589.

3. Stoyanov SR, Hides L, Kavanagh DJ et al. (2015) JMIR mHealth uHealth 3(1), e27. 